\title{
The interval count of interval graphs and orders: a short survey
}

\author{
Márcia R. Cerioli • Fabiano de S. Oliveira • \\ Jayme L. Szwarcfiter
}

Received: 4 October 2011 / Accepted: 31 October 2011 / Published online: 26 November 2011

(C) The Brazilian Computer Society 2011

\begin{abstract}
The interval count problem determines the smallest number of interval lengths needed in order to represent an interval model of a given interval graph or interval order. Despite the large number of studies about interval graphs and interval orders, surprisingly only a few results on the interval count problem are known. In this work, we provide a short survey about the interval count and related problems. a graph and the number of its maximal cliques.
\end{abstract}

Keywords Interval count $\cdot$ Interval lengths $\cdot$ Number of lengths

\section{Introduction}

The interest in interval graphs and orders comes from both their central role in many applications and purely theoretical questions $[15,17,29]$. They potentially arise in applications

M.R. Cerioli · F. de S. Oliveira ( $₫)$ · J.L. Szwarcfiter

COPPE, Universidade Federal do Rio de Janeiro (UFRJ),

Caixa Postal 68511, 21941-972 Rio de Janeiro, RJ, Brazil

e-mail: fabianoo@gmail.com

M.R. Cerioli

e-mail: cerioli@cos.ufrj.br

M.R. Cerioli · J.L. Szwarcfiter

Instituto de Matemática, Universidade Federal do Rio de Janeiro (UFRJ), Caixa Postal 68530, 21941-972 Rio de Janeiro, RJ,

Brazil

\section{J.L. Szwarcfiter}

Núcleo de Computação Eletrônica, Universidade Federal do Rio de Janeiro (UFRJ), Caixa Postal: 2324, 20010-974 Rio de Janeiro, RJ, Brazil

e-mail: jayme@nce.ufrj.br for which there are events associated to time intervals corresponding to the duration of the events. Among such applications, as discussed in [27], there are those related to planning [2], scheduling [26], archeology [22], temporal reasoning [1], medical diagnosis [25], and circuit design [33]. Furthermore, there are applications not related directly to duration of events in the fields of genetics [3], physical mapping of DNA [7, 21], and behavioral psychology [10].

An interval model is a family of closed intervals of the real line. A graph $G$ is an interval graph if there exists an interval model $\mathcal{R}=\left\{I_{v} \mid v \in V(G)\right\}$ such that for all distinct $x, y \in V(G), I_{x} \cap I_{y} \neq \emptyset$ if and only if $x y \in E(G)$. Figure 1 presents an interval graph and a corresponding interval model.

An $\operatorname{order}(X, \prec)$ is a transitive and irreflexive binary relation $\prec$ on $X$. An order $P=(X, \prec)$ is an interval order if there exists an interval model $\mathcal{R}=\left\{I_{x} \mid x \in X\right\}$ such that for all $x, y \in X, x \prec y$ if and only if $I_{x}$ precedes (is entirely to the left of) $I_{y}$. In these cases, $\mathcal{R}$ is an interval model of $G$ (resp. $P$ ). Besides, we say that $G$ (resp. $P$ ) is the graph (resp. order) corresponding to $\mathcal{R}$.

For some given graph $G$ (resp. order $P$ ), we consider the problem of computing the smallest number $I C(G)$ (resp. $I C(P))$ of interval lengths for an interval model of $G$ (resp. $P$ ), named in both cases the interval count problem [15, 24]. Figure 2 presents another interval model of the graph defined in Fig. 1 in which there are only two interval lengths. Clearly, the minimum number of lengths required for any interval model of this graph is two, which is, therefore, the interval count of this graph.

The importance of the interval count is evident. Since the definition of interval graphs needs the construction of intervals, it is a natural question to determine the minimum number of lengths of intervals required for a representation. Furthermore, interval count is related to a number of 
Fig. 1 Interval graph and one of its interval models
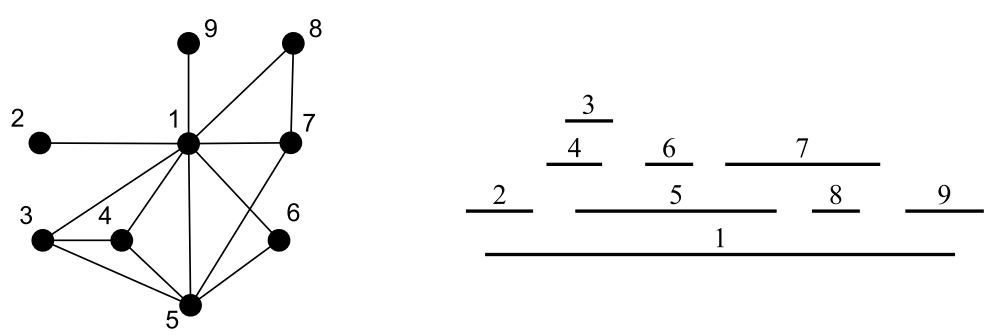

Fig. 2 Two lengths of intervals is both needed and sufficient
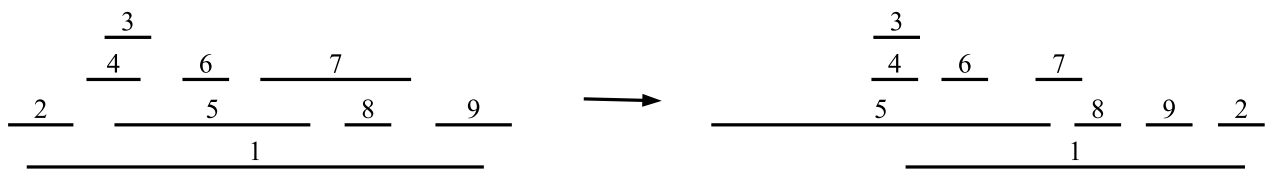

practical applications. The outputs of interest among the applications that require the construction of interval models can be constrained by a desirable number of distinct interval lengths, generally as a matter of convenience or fairness. For instance, the problem of allocating initial and final hours for presentations at a workshop could require the generation of only two durations for presentations (one for the ordinary speakers, another for the invited speakers). Another example of real-world application is the scheduling of class periods at a university. A period is a certain time (say, one hour) of which the duration of any particular class should be a multiple. Some individual classes of a discipline may require several consecutive periods to be delivered, whereas for other classes a single period suffices. Each discipline requires a certain amount of periods to be delivered. If the scheduling of disciplines is carried out in such a way that each period of a discipline is individually planned, then the scheduling algorithm must assign consecutive periods for some classes. In order to avoid dealing with dependencies among periods, we can redefine the total duration required for each class as a period. In this approach, each class is delivered in only one period of variable length. Moreover, the interval graph to be generated as a result of the scheduling process is known to have a certain interval count by construction (which is, the total number of distinct required class lengths).

The interval count problem was suggested by Ronald Graham (cf. [24]). There are graphs having arbitrary interval count values. In particular, let $G_{1}$ be the graph consisting of a single vertex $u_{1}$. For each $k \geq 2$, let $G_{k}$ be the graph consisting of three disjoint copies of $G_{k-1}$ plus a vertex $u_{k}$ adjacent to all others. Clearly, $G_{k}$ is an interval graph. Furthermore, it is easy to show that $\operatorname{IC}\left(G_{k}\right)=k$ for each $k \geq 1$. The complexity of deciding whether $I C(G)=k$ (resp. $I C(P)=k$ ) for a graph $G$ (resp. order $P$ ) and an integer $k>1$ is open: it is not known presently whether it is an NPcomplete problem.

The interval count problem is an intriguing problem, in which very intuitive statements are proved not to hold. As an example, Graham stated the conjecture that the interval count of a graph decreases by at most one unit when exactly one vertex is removed. Intuitively speaking, if a graph has an interval model requiring at least $k$ different interval lengths, the operation of removing one interval from this model (or, equivalently, a vertex of this graph) seems not to result in a graph which has an interval model using $k-2$ or less different interval lengths. This conjecture was proved to hold only for certain interval graphs, as we discuss in the next sections.

Since the interval count problem is defined only for interval graphs (resp. orders), it is assumed that the graphs (resp. orders) have an associated interval model when this problem is considered. Denoting by $I C(\mathcal{R})$ the number of distinct lengths of a given interval model $\mathcal{R}$, for a given order $P$, we can write

$I C(P)=\min \{I C(\mathcal{R}) \mid \mathcal{R}$ is an interval model of $P\}$

and, similarly, given a graph $G$,

$I C(G)=\min \{I C(\mathcal{R}) \mid \mathcal{R}$ is an interval model of $G\}$.

When there exists an interval model $\mathcal{R}$ which is an interval model of both an order $P$ and a graph $G$, we say that $P$ agrees with $G$. Note that an interval order agrees with a unique interval graph, but the converse is false: an interval graph may have exponentially many interval orders agreeing with it. Using this relation of agreement, we can enunciate the interval count of graphs in a way which evidences its relationship to the interval count of orders. Given a graph $G$, we can write that:

$I C(G)=\min \{I C(P) \mid P$ agrees with $G\}$.

In the literature, there exist a collection of results on the interval count of graphs and orders. In this paper, we present them in the version as they were originally stated, concerning the interval count of either orders, or graphs, or both. Note that when a result is presented only for the interval count of graphs (resp. orders), the counterpart result for the 
interval count of orders (resp. graphs) does not necessarily hold.

Denote the left and right extreme points of an interval $I$ by $\ell(I)$ and $r(I)$, respectively. When an interval model of an interval graph (resp. interval order) is clear in the context, for convenience, we may use the concepts of vertex (resp. element) and its corresponding interval interchangeably. A unit interval graph is an interval graph which admits an interval model whose intervals are unitary length. A $K_{1, r}$ graph is the complete bipartite graph in which the cardinality of the sets forming the bipartition are 1 and $r$. A $(1+3)$ order is isomorphic to the order $(\{a, b, c, d\}, \prec)$ such that $b \prec c \prec d$ and $a$ is incomparable to $b, c$, and $d$. A proper interval graph is an interval graph which admits an interval model for which there do not exist intervals $I_{x}$ and $I_{y}$ such that $\ell\left(I_{x}\right)<\ell\left(I_{y}\right)<r\left(I_{y}\right)<r\left(I_{x}\right)$. Finally, if the adjacent vertices to a given vertex $v$ is a clique, then $v$ is called a simplicial vertex.

In Sects. 2, 3, and 4, we provide a brief survey about the interval count of graphs and orders which have respectively interval count one, interval count two, and arbitrary interval count values. In Sect. 5, we present solutions of the interval count problem when the graphs and orders are restricted to belong to certain subclasses of interval graphs and interval orders. In Sect. 6, we show a relation between the interval count of a graph and the number of its maximal cliques, providing a tight upper bound for the interval count of graphs having $q$ cliques. In Sect. 7, we present related problems which are also concerned about the interval lengths, but not about the minimization of the number of them. Finally, in Sect. 8, we provide concluding remarks on the interval count problem.

For the omitted notation in this paper, refer to [5] for general graph theory, [32] for general order theory, and [15, 17] for a specialized discussion about interval graphs and interval orders.

\section{Interval count one}

The question of deciding whether $I C(G)=1$ for an interval graph $G$ is equivalent to that of recognizing whether $G$ is a unit interval graph. In fact, given an interval model using only intervals of the same length, it is possible to either compress or expand proportionally all intervals so that they are transformed into unit intervals. The recognition problem of unit interval graphs is well known since the sixties [28] and can be solved by polynomial-time algorithms, some of them being of linear time [11-14, 16, 19, 28]. Moreover, unit interval graphs are characterized by a single finite forbidden structure, as stated in Theorem 1 (firstly obtained by [28]).

Theorem 1 ([4]) If $G$ is an interval graph, then $G$ is a unit interval graph if and only if $G$ is $K_{1,3}$-free.
Proof Clearly a $K_{1,3}$ graph cannot be represented by a unit interval model. Conversely, suppose that $G$ is $K_{1,3}$-free. We claim that there exists an interval model of $G$ in which no interval is included in another interval. By way of contradiction, suppose that the claim is false. Let $\mathcal{R}=\left\{I_{v} \mid v \in\right.$ $V(G)\}$ be the interval model having the fewest number of interval inclusions, and let $I_{x} \subseteq I_{y}$ for some $x, y \in V(G)$. Since $\ell\left(I_{x}\right)$ cannot be moved to the left passing through $\ell\left(I_{y}\right)$, by the minimality of the number of interval inclusions, then there exists $a \in V(G)$ such that $I_{a} \cap I_{y} \neq \emptyset$ and $r\left(I_{a}\right)<\ell\left(I_{x}\right)$. Symmetrically, there exists $b \in V(G)$ such that $I_{b} \cap I_{y} \neq \varnothing$ and $r\left(I_{x}\right)<\ell\left(I_{b}\right)$. But then $G[\{y, a, x, b\}]$ is isomorphic to a $K_{1,3}$, a contradiction. Therefore, let $\mathcal{R}=$ $\left\{I_{v} \mid v \in V(G)\right\}$ be an interval model of $G$ having no interval inclusions. We build a unit interval model of $G$ iteratively transforming $\mathcal{R}$ as follows.

In each iteration, let $I$ be the nonunit interval having the leftmost left interval extreme point. If there is no right extreme point contained in the portion $(\ell(I), r(I))$ of the model, then let $p=\ell(I)$. Otherwise, let $p$ be the largest such an extreme point (in this case, $p$ would belong to an interval that has already been adjusted to have unitary length, in order to not contradict both the fact of $\mathcal{R}$ having no interval inclusion and the choice of $I$ ). Thus, $p \leq$ $\min \{\ell(I)+1, r(I)\}$. Adjust the model by compressing or expanding proportionally the portion of the model defined by $[p, r(I)]$ so that it fits in the portion $[p, \ell(I)+1]$ of the model (translate the portion $[r(I), \infty)$ of the model to $[\ell(I)+1, \infty))$. The order of interval extreme points does not change, intervals iterated earlier than $I$ remains unitary length, and now $I$ has also length 1. Clearly, when no more intervals can be selected as $I$, the resulting interval model is a unit interval model of $G$.

Corollary 2 ([11-14, 16, 19, 28]) If $G$ is an interval graph, then $G$ is a unit interval graph if and only if $G$ is a proper interval graph.

Proof Clearly, a unit interval graph is a proper interval graph. Conversely, the proof of Theorem 1 transforms a proper interval model into a unit interval model.

Corollary 3 ([28]) If $P$ is an interval order, then $P$ is a unit interval order if and only if $P$ has no induced $(1+3)$-order.

Proof It follows from Theorem 1 and the fact that every order which agrees with a $K_{1,3}$ graph is a $(1+3)$-order.

Therefore, having interval count one for graphs and orders is a well-solved question. Moreover, note that Graham's conjecture holds trivially for all graphs having interval count one. 


\section{Interval count two}

Although deciding whether a graph or order has interval count one is a well-known and well-solved problem, having linear-time recognition algorithms, it is not known whether the complexity of deciding if $I C(G)=2(\operatorname{resp} . I C(P)=2)$ for a graph $G$ (resp. order $P$ ) is a polynomial-time solvable problem.

Despite the lack of an efficient procedure to recognize whether the interval count of a graph is at most two, Skrien characterized those, among such graphs having interval count two, that admit a two-length model where the shortest size is equal to zero [30]. In other words, Skrien characterized the graphs which can be represented by a model in which every length is either zero or one. Obviously, if $G$ is a graph which admits a model like this, the set $S$ of vertices having length equal to zero is a set of simplicial vertices. Furthermore, the graph $G[V(G) \backslash S]$, induced from $G$ by the vertices having length equal to one, is a unit interval graph. Conversely, the existence of a set $S$ of simplicial vertices such that $G[V(G) \backslash S]$ is a unit interval graph does not suffice to state that there is a model of $G$ having only zero or one interval length, as exemplified in [30]. The conditions for sufficiency is stated as follows.

Theorem 4 ([30]) Let $G$ be an interval graph, and $S$ be a set of simplicial vertices of $G$. Such a graph $G$ can be represented by a model having only zero or one interval length if and only if there are orientations $O$ of $G \backslash S$ and $F$ of $\bar{G}$ such that:

1. $O \cup F$ is a transitive orientation.

2. $O F \cup F O \cup F F \subset F$ (where $A B$ denotes the set of pairs $a b$ such that $a x \in A$ and $x b \in B$ ).

The conditions of this characterization can be tested in $O\left(n^{3}\right)$ time (cf. [30]).

Fishburn investigated the topology of the models of orders in $\mathcal{P}_{2}$, the class of orders having interval count equal to 2. Given an order $P \in \mathcal{P}_{2}$, it is clear that there exist interval models of $P$ having the smallest of the two distinct interval lengths equal to one. The question was to determine the set $\theta(P)$ of admissible lengths for the greatest length. In other words, given an interval order $P=(X, \prec)$, the problem is to determine the set

$\theta(P)=\{\alpha>1 \mid$ there exists an interval model

$$
\begin{aligned}
& R=\left\{I_{x} \mid x \in X\right\} \text { of } P \text { having } \\
& I C(\mathcal{R})=2 \text { such that }\left|I_{x}\right| \in\{1, \alpha\} \\
& \text { for each } x \in X\}
\end{aligned}
$$

As an example, if $\mathcal{R}$ is an interval model of the graph $K_{1, t+2}, t \geq 1$, and $P$ is the corresponding interval order

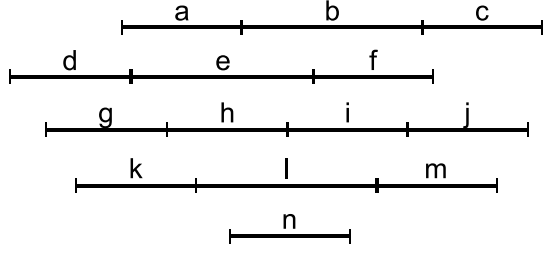

Fig. 3 Example of order $P$ having $\theta(P)=(1,2)$

to $\mathcal{R}$, then $\theta(P)=(t, \infty)$. In a first glance, $\theta(P)$ seems to be connected. The longer lengths in a two-length interval model seem to allow their incremental increase without affecting the two-length representation of the model, in a similar way that a $K_{1,3}$ 's model does. Fishburn proved that, for some orders, such an increase on the longer length has a limit. He presented examples of orders $P \in \mathcal{P}_{2}$ such that $\theta(P)=(1, k)$ for each $k \geq 2$. We present such an example for $k=2$ below. Examples for $k>2$ can be found in [15].

Theorem 5 ([15]) If $P$ is the corresponding order to the interval model depicted in Fig. 3, then $\theta(P)=(1,2)$.

Proof Let $\left\{I_{x} \mid x \in X\right\}$ be a two-length interval model of $P$ having the shorter size equal to 1 , and let $\alpha \in \theta(P)$ be the longer length. Due to the suborders induced by the sets $\{d, e, f, h\},\{a, b, c, i\}$, and $\{k, l, m, n\}$, it follows that $\left|I_{h}\right|=\left|I_{i}\right|=1$ and $\left|I_{l}\right|=\alpha$ (in Fig. 3, note that the right extreme points of intervals coincide with the left extreme points of succeeding intervals drawn in the same line; for example, $r\left(I_{a}\right)=\ell\left(I_{b}\right), r\left(I_{b}\right)=\ell\left(I_{c}\right), r\left(I_{g}\right)=\ell\left(I_{h}\right)$, and so on). Clearly, $\alpha<2$. Moreover, it is easy to check that the interval model of Fig. 3 can be adjusted so that every $1<\alpha<2$ is admissible, by changing the size of the longer intervals $b, e, l$ (in Fig. $3, \alpha=1.5$ ).

In [31], it is conjectured that $\theta(P)$ would be an open interval. However, Fishburn also presented orders $P \in \mathcal{P}_{2}$ such that $\theta(P)=(2-1 / k, 2) \cup(k, \infty)$ for each $k \geq 2$, which means that, surprisingly, $\theta(P)$ can be a disconnected set. Furthermore, he proved that for each $k \geq 2$, there exists $P \in \mathcal{P}_{2}$ such that $\theta(P)$ is the union of $k$ distinct open intervals.

Regarding the Graham's conjecture, all graphs having interval count two trivially satisfy it. Furthermore, Leibowitz, Assmann, and Peck [24] characterized another case for which the conjecture holds: if $G$ is a graph such that $I C(G \backslash x)=1$ for some vertex $x$ of $G$, then $I C(G) \leq 2$.

\section{Arbitrary interval count}

Just as the complexity of deciding the existence of an interval model using two interval lengths, it is also unknown 
Fig. 4 Definition of order $Q_{r}$, for each $r \geq 3$

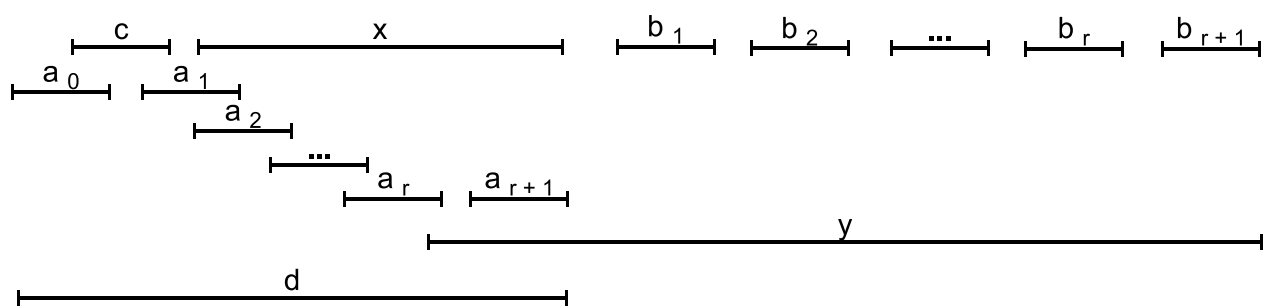

whether deciding if the interval count of graph is $k$, for an integer $k>1$, is an NP-complete problem.

Since having interval count one has a simple characterization in terms of forbidden induced subgraphs, a natural approach is to investigate characterizations of graphs (resp. orders) of arbitrary interval count values by forbidden induced subgraphs (resp. suborders) as well. In [15], it is shown that the list of forbidden suborders to characterize the orders which have interval count equal to $k \geq 2$ is infinite. A similar result holds for graphs.

Theorem 6 ([15]) If $\mathcal{S}$ is the set of orders such that, given an interval order $P$ and $k \geq 2, I C(P) \leq k$ if and only if $P$ has no induced suborder isomorphic to one of those in $\mathcal{S}$, then $\mathcal{S}$ is infinite.

Proof First interval orders $P=(X, \prec)$ of arbitrary large finite cardinality which have $I C(P)>2$ whose every proper induced suborders $P^{\prime}$ have $I C\left(P^{\prime}\right)=2$ are constructed, setting the result for $k=2$. Then the result is extended for the case $k>2$.

Let $P=Q_{r}$ be the order defined in Fig. 4 for each $r \geq 3$. If $I C\left(Q_{r}\right)=2$, then $a_{1}, \ldots, a_{r+1}, b_{1}, \ldots$, and $b_{r}$ must have a shorter length. But then $x$ and $y$ could not have the same length since, representing the shorter length by $\beta, y$ 's left extreme point is within $r \beta$ units of $x$ 's left extreme point, whereas $y$ 's right extreme point is more than $r \beta$ units greater than $x$ 's right extreme point. Therefore, $I C\left(Q_{r}\right)>2$.

To prove that every proper induced suborder $Q^{\prime}$ of $Q_{r}$ has $\operatorname{IC}\left(Q^{\prime}\right)=2$, it suffices to consider only induced suborders obtained by removing one element from $X$ as follows:

1. $Q^{\prime}=Q_{r} \backslash x$ : Clearly there exists an interval model having $d$ and $y$ as the longer length and the remaining intervals with the shorter.

2. $Q^{\prime}=Q_{r} \backslash y: d$ and $x$ having the longer length and the remaining intervals with the shorter.

3. $Q^{\prime}=Q_{r} \backslash d$ : All, but $a_{1}, x$, and $y$, with the shorter length; the longer length for $a_{1}$ allows $x$ and $y$ to have the same longer length.

4. $Q^{\prime}=Q_{r} \backslash c$ : All, but $d, x$, and $y$, with the shorter length; begin $a_{1}$ to the right of the left extreme point of $x$ so that $x$ and $y$ can have the same longer length.

5. $Q^{\prime}=Q_{r} \backslash a_{0}$ : All, but $a_{1}, d, x$, and $y$, with the shorter length.
6. $Q^{\prime}=Q_{r} \backslash a_{i}$, for some $1 \leq i \leq r$. This leaves a gap in the path $a_{1}, \ldots, a_{r}$, and consequently there is a point of $x$ which belongs only to $x$ and $d$. Therefore, clearly there exists an interval model in which all, but $d, x$, and $y$, have the shorter length.

7. $Q^{\prime}=Q_{r} \backslash a_{r+1}$ : All, but $a_{r}, d, x$, and $y$, with the shorter length. A possible model is the following: $I_{c}=[-1,0]$, $I_{a_{0}}=[-1-\lambda,-\lambda], I_{a_{i}}=[i-1, i]$ for each $1 \leq i \leq r-$ $1, I_{b_{i}}=[r-2+i+N+i \lambda, r-1+i+N+i \lambda]$ for each $1 \leq i \leq r+1, I_{x}=[\lambda, N+\lambda], I_{y}=[N-\lambda, 2 N-\lambda]$, $I_{d}=[-\lambda, N-\lambda], I_{a_{r}}=[r-1, r-1+N]$. Such a model clearly suffices when $\lambda>0$ is small and $N$ is large.

8. $Q^{\prime}=Q_{r} \backslash b_{i}$ for some $1 \leq i \leq r$. For convenience, renumber the elements $\left\{b_{1}, \ldots, b_{r+1}\right\} \backslash\left\{b_{i}\right\}$ as $b_{1}, \ldots, b_{r}$ following the original order. Use the preceding model for $x, c, a_{0}, \ldots$, and $a_{r-1}$ along with $I_{a_{i}}=[i-1, i]$ for each $r \leq i \leq r+1, I_{b_{i}}=[N-1+i+(i+1) \lambda, N+$ $i+(i+1) \lambda]$ for each $1 \leq i \leq r, I_{y}=[r, r+N], I_{d}=$ $[r+1-N, r+1]$. Again, this model suffices when $\lambda>0$ is small and $N$ is large. Furthermore, for $y$ to intersect $b_{r}$, we need $r+N \geq N-1+r+(r+1) \lambda$, or $\lambda \leq 1 /(r+1)$.

Cases 1 through 8 account for all elements in $X$. Hence, for each proper induced suborder $Q^{\prime}$ of $Q_{r}$, it holds that IC $\left(Q^{\prime}\right) \leq 2$.

To prove the result for $k \geq 3$, assume by way of contradiction that, for some $k \geq 3$, there exists a finite set $\mathcal{S}$ of interval orders such that $I C(P) \leq k$ if and only if $P$ has no induced suborder isomorphic to one of those in $\mathcal{S}$ for every interval order $P$. The reasoning below shows that the same would hold for $k-1$ in place of $k$. Applying the claim successively, it follows that the same would hold for $k=2$, a contradiction.

Note that $I C(Q)>k$ for each $Q \in \mathcal{S}$. Let $\mathcal{S}^{\prime}$ consist of all induced suborder $Q^{\prime}$ of $Q \in \mathcal{S}$ such that $\operatorname{IC}\left(Q^{\prime}\right) \geq k$. Clearly, $\mathcal{S} \subseteq \mathcal{S}^{\prime}$, and $\mathcal{S}^{\prime}$ is finite. It will be proved that $\mathcal{S}^{\prime}$ is a finite set for $k-1$ as $\mathcal{S}$ is for $k$.

Let $R=\left(X_{R}, \prec_{R}\right)$ be an order such that $I C(R) \geq k$. If $I C(R)>k$, then a proper induced suborder of $R$ is in $\mathcal{S} \subseteq \mathcal{S}^{\prime}$. Assume therefore that $I C(R)=k$ and let $R^{\prime}$ be the interval order obtained from $R$ by adding an element $a$ preceding each element in $X_{R}$, an element $b$ succeeding each element in $X_{R}$, and an element $c$ incomparable to both $a$ and $b$ (and, therefore, incomparable to each element in $\left.X_{R}\right)$. Clearly, $I C\left(R^{\prime}\right)=k+1$, and therefore there 
Fig. 5 For the graph $G$ above, $I C(G)=I C(G \backslash x)+2$

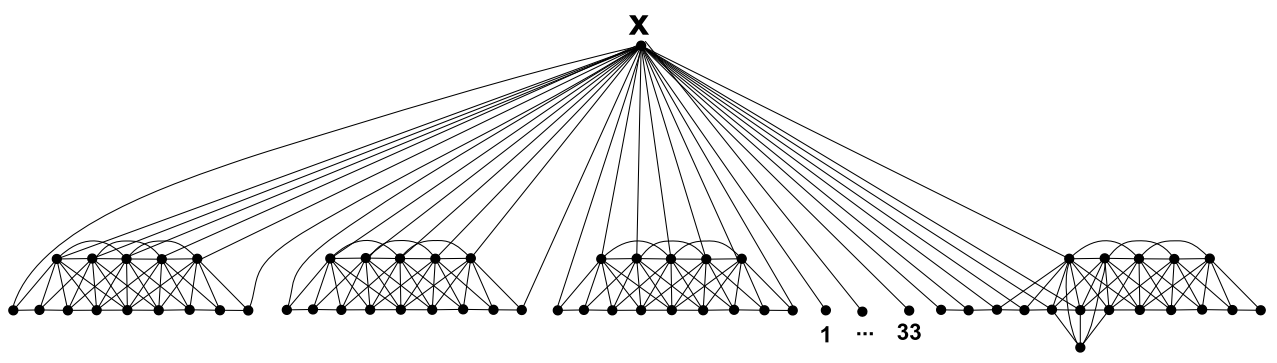

is a proper induced suborder $R^{\prime \prime}$ of $R^{\prime}$ such that $R^{\prime \prime} \in \mathcal{S}$. Note that $R^{\prime \prime} \backslash\{a, b, c\}$ is an induced suborder of $R$. Besides, $R^{\prime \prime}$ must contain $c$, or $R^{\prime \prime}$ could be representable by $k$ lengths, a contradiction. In addition, $I C\left(R^{\prime \prime} \backslash\{c\}\right)=$ $I C\left(R^{\prime \prime} \backslash\{a, b, c\}\right) \leq I C(R)=k$, and $I C\left(R^{\prime \prime} \backslash\{a, b, c\}\right)$ cannot be less than $k$, or otherwise $I C\left(R^{\prime \prime}\right)$ would not be $k+1$. Since $I C\left(R^{\prime \prime} \backslash\{a, b, c\}\right)=k, R^{\prime \prime} \backslash\{a, b, c\}$ is in $\mathcal{S}^{\prime}$. The claim follows as a result.

In contrast to the previous sections, Graham's conjecture does not necessarily hold for graphs having arbitrary interval count values. Leibowitz, Assmann, and Peck [24] presented examples of graphs having $I C(G)>2$ for which $I C(G)=$ $I C(G \backslash x)+2$ given a particular vertex $x$ of $G$. Figure 5 depicts one of such examples.

This result refutes the conjecture, but it makes room for a generalized version of it, which we pose here: does there exist some integer $k>1$ for which it holds that the interval count of a graph decreases by at most $k$ when exactly one vertex is removed? In [31], it is conjectured that the removal of a vertex may decrease the interval count by an arbitrary large amount.

Fishburn also considered extremal problems on the interval count of an order, its number of elements, and its number of maximal antichains. Given a graph $G$ (resp. order $P$ ), denote by $q(G)$ (resp. $q(P)$ ) the number of its maximal cliques (resp. maximal antichains). Consider the following functions:

$\sigma(k)=\min \{|X| \mid P=(X, \prec)$ is an order and $I C(P) \geq k\} ;$

and

$$
\begin{gathered}
v(k, q)=\min \{|X| \mid P=(X, \prec) \text { is an order, } q(P)=q, \\
I C(P) \geq k\}
\end{gathered}
$$

Obviously, $\sigma(k)$ is equal to the minimum of $v(k, q)$ varying $q$ over its domain. Particularly, Fishburn showed that $\sigma(k)=\min \{v(k, q) \mid q \geq 2 k-1\}$. The restriction $q \geq 2 k-1$ follows from the fact that the function $v(k, q)$ is undefined for each $k>\lfloor(q+1) / 2\rfloor$, i.e., $I C(P)<k$ for all $k>\lfloor(q+1) / 2\rfloor$ and order $P$ such that $q(P)=q$. Besides, he proved that $v(k, q) \leq k+q-1$ holds in general and calculated the exact value of the function $v(k, q)$ when $k$ and $q$ are restricted to some specific values. In Sect. 6, we show that for any graph $G$, it holds that $\max \{I C(G) \mid q(G)=$ $q\}=\lfloor(q+1) / 2\rfloor$, which implies the domain of the function $v(k, q)$. We provide a simpler proof than that from [15].

\section{Interval count of subclasses of graphs and orders}

In this section we present results which come from the investigation of the interval count problem restricted to certain subclasses of interval graphs and interval orders.

Let $P_{n}$ be an induced path on $n$ vertices. Let $G$ be a graph, and $v \in V(G)$. The neighborhood of $v$ is the set $N(v)=\{w \mid(v, w) \in E(G)\}$. The substitution of $v$ by the graph $G^{\prime}$ is the graph $H$ obtained from the disjoint union $(G \backslash v) \cup G^{\prime}$ plus the edges $u w$ such that $u \in N(v)$ and $w \in V\left(G^{\prime}\right)$. In such a case, we say that $H$ is obtained from $G$ by substituting $v$ by $G^{\prime}$.

A graph is a tree if it is connected and acyclic. A graph is threshold if its vertex set can be partitioned into $K \cup I$ such that $K$ is a clique, $I$ is an independent set, and there exists an ordering $v_{1}, \ldots, v_{|I|}$ of the vertices of $I$ such that $N\left(v_{i}\right) \subseteq N\left(v_{i+1}\right)$ for each $1 \leq i<|I|$ (or, equivalently, there exists an ordering $u_{1}, \ldots, u_{|K|}$ of the vertices of $K$ such that $I \cap N\left(u_{i+1}\right) \subseteq I \cap N\left(u_{i}\right)$ for each $\left.1 \leq i<|K|\right)$. A graph $G$ is almost $K_{1,3}$-free if there exists $v \in V(G)$ such that $G \backslash v$ is $K_{1,3}$-free. A graph $G$ is starlike-threshold if it can be obtained from a threshold graph substituting each vertex of the independent set by a corresponding clique. An interval graph is trivially perfect (TP) if it is $P_{4}$-free [17].

A graph is generalized-threshold if it can be obtained from a threshold graph by substituting each vertex of the independent set by a corresponding unit-interval graph. An $X F_{1}^{n}$ graph $(n \geq 0)$ consists of a path $P$ of length $n+2$ and a vertex that is adjacent to every vertex of $P$ except its extremes [6]. Therefore, $X F_{1}^{0}$ is a $K_{1,3}$, and $X F_{1}^{1}$ is a bull. For convenience, in this paper we call the graph $X F_{1}^{n}$ for each $n \geq 1$ an extended-bull. The extended-bull graph is depicted in Fig. 6. A graph is extended-bull-free if it has no extendedbull as an induced subgraph.

Figure 7 presents the inclusion diagram among these graph classes. In this diagram, a class $\mathcal{A}$ is a generalization of a class $\mathcal{B}$ precisely when there exists a top-down path from $\mathcal{A}$ to $\mathcal{B}$. Each class in the figure is labeled by a graph 
belonging to this class, which is a separating example, that is, which shows that this class is distinct (either proper containing, or proper being contained, or not having a generalization relationship) from each other class. In those examples, a graph $G$ having an edge $u v$ where $v$ is represented by a white vertex labeled with $G^{\prime}$ represents the graph obtained from $G$ substituting $v$ by $G^{\prime}$.

In [23], it is proved that the interval count of trees, threshold graphs and almost- $K_{1,3}$-free graphs is at most 2 . In [8], it is observed that the starlike-threshold graphs also have interval count at most 2. Recently, Cerioli, Oliveira, and Szwarcfiter [9] extended the result of having the interval count limited to 2 to generalized-threshold graphs. Furthermore, in [9], it has been described polynomial-time algorithms to compute the interval count of extended-bull-free graphs (and, in particular, trivially perfect graphs). Such a class contains instances of graphs with arbitrary interval count values.

To our knowledge, there are no other subclasses of interval graphs and orders for which it is currently known how to compute their interval count efficiently.

Fig. 6 The extended-bull graph, for $n \geq 1$

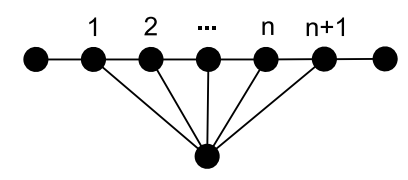

\section{Relation to the number of maximal cliques}

Obviously, $I C(G) \leq|V(G)|$ for any graph $G$, which consists in a trivial upper bound on the interval count of a graph. It is possible however to derive better upper bounds. For instance, consider the following observation. Let $\mathcal{R}$ be an interval model of the graph $G$ such that $I C(\mathcal{R})=I C(G)$. Reading the maximal cliques of $G$ from left to right in $\mathcal{R}$, note that there exists an interval $I_{1}$ belonging exclusively to the first maximal clique, or otherwise the first maximal clique would be a subset of the second one. By a similar argumentation, there exists an interval $I_{q}$ belonging exclusively to the last maximal clique. Since there is no reason to have $I_{1}$ and $I_{q}$ with distinct lengths, we have $I C(G) \leq$ $|V(G)|-1$ for any graph $G$.

In this section, we address an extremal problem concerning the interval count of graphs having a fixed number of maximal cliques. For any $q \geq 1$, let $f(q)=\max \{I C(G) \mid$ $q(G)=q\}$, where $q(G)$ denotes the number of maximal cliques of $G$.

Theorem 7 For each $q \geq 1, f(q)=\lfloor(q+1) / 2\rfloor$.

Proof The result is clear when $q \leq 2$, since such graphs are $K_{1,3}$-free. Therefore, assume that $q>2$. For each $i \geq 1$, let $G_{i}$ be the graph defined schematically in Fig. 8. The double lines linking $u_{i}$ to $H_{i-1}$ mean that the vertex $u_{i}$ is adjacent to all vertices of the induced subgraph $H_{i-1}$.

Fig. 7 Inclusion diagram

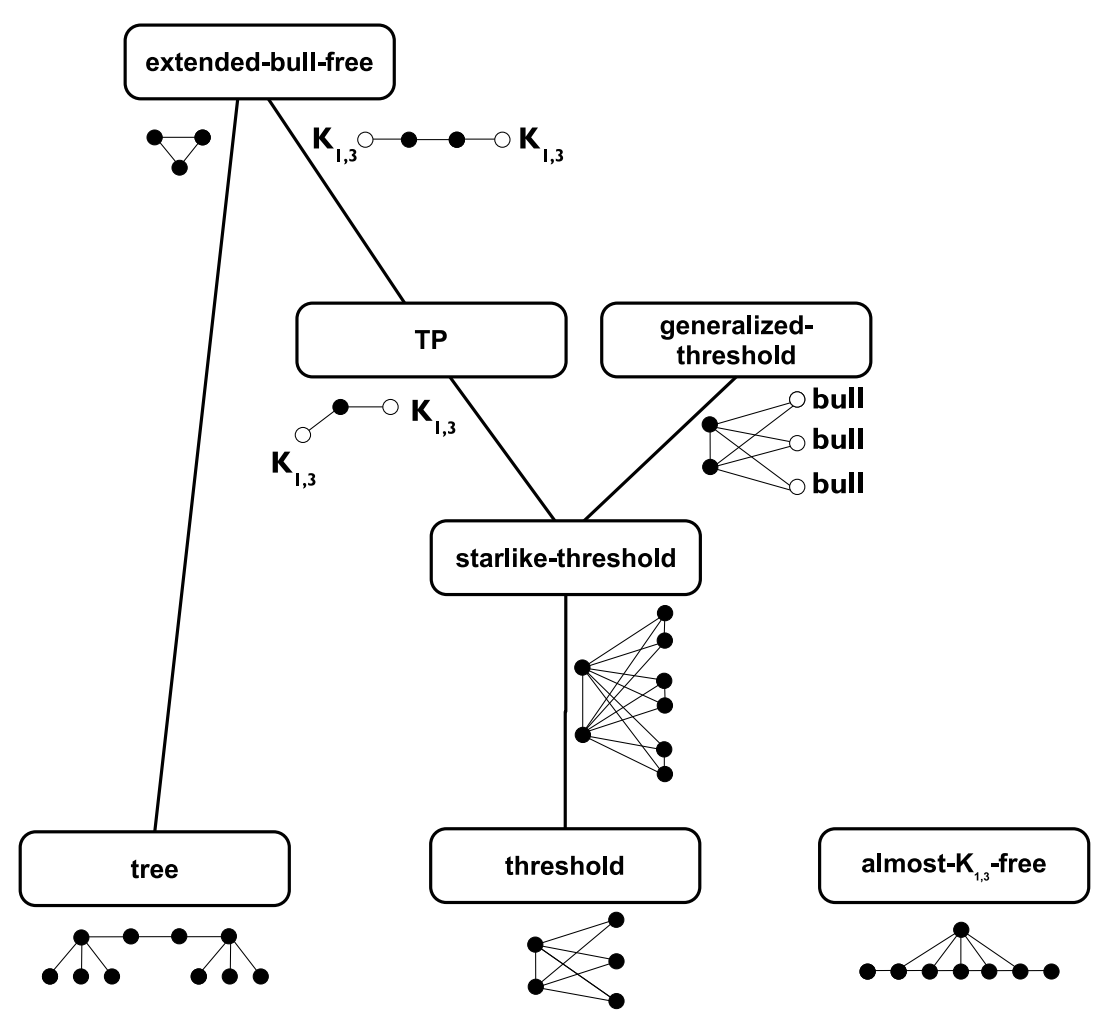


Fig. 8 Auxiliary graphs $H_{k}$, $k \geq 0$, and $G_{i}, i \geq 1$

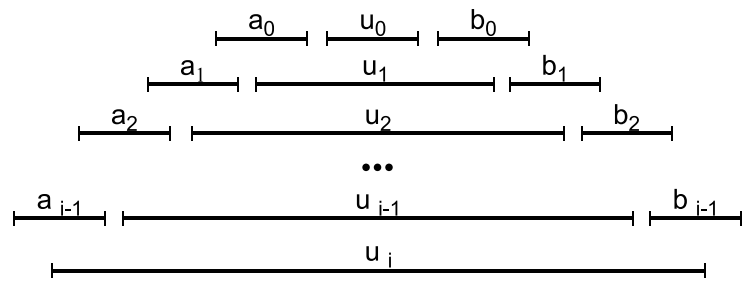

Fig. 9 One of the interval models of the graph $G_{i}, i \geq 1$

For each $i \geq 1$, the number of maximal cliques of $G_{i}$ can be easily worked out according to its definition as being $q\left(G_{i}\right)=2 i+1$. Moreover, for any interval model $\left\{I_{v} \mid v \in\right.$ $\left.V\left(G_{i}\right)\right\}$ of $G_{i},\left|I_{u_{k}}\right|>\left|I_{u_{k-1}}\right|$ for each $1 \leq k \leq i$. Thus, $I C\left(G_{i}\right) \geq i+1$. As a matter of fact, $\operatorname{IC}\left(G_{i}\right)=i+1$ as it can be verified in the interval model of $G_{i}$ depicted in Fig. 9.

Let $G$ be either the graph $G_{(q-1) / 2}$ if $q$ is odd or the graph $G_{(q-2) / 2}$ plus an isolated vertex, otherwise. Therefore, $f(q) \geq I C(G)=\lfloor(q+1) / 2\rfloor$.

On the other hand, let $G$ be a graph with $q$ maximal cliques. We show an upper bound for $f(q)$ by describing an algorithm which builds an interval model $\mathcal{R}$ of $G$ such that $I C(\mathcal{R}) \leq\lfloor(q+1) / 2\rfloor$. Since $G$ is a general graph with $q$ maximal cliques, we have $f(q) \leq\lfloor(q+1) / 2\rfloor$.

Let $C_{1}, \ldots, C_{q}$ be the maximal cliques read from left to right of some interval model of $G$, and let $m=\lfloor(q+1) / 2\rfloor$. Initially, assume that all intervals in the cliques $C_{m}$ or $C_{m+1}$ are unit length. Then, on each step $i=1, \ldots, m-1$, move each left extreme point of the intervals in $C_{m-i} \cap C_{m-i+1}$ to the left and move each right extreme point of the intervals in $C_{m+i} \cap C_{m+i+1}$ to the right in such a way that the following two conditions hold: (i) the modified intervals have the greatest length, and (ii) there exist two points $p_{1}<p_{2}$ such that the sets of intervals which contain $p_{1}$ and $p_{2}$ are $C_{m-i} \cap C_{m-i+1}$ and $C_{m+i} \cap C_{m+i+1}$, respectively. For each $v \in C_{m-i} \backslash C_{m-i+1}$, add $I_{v}$ such that $\left|I_{v}\right|=1$ and $r\left(I_{v}\right)=p_{1}$. Symmetrically, for each $v \in C_{m+i+1} \backslash C_{m+i}$, add $I_{v}$ such that $\left|I_{v}\right|=1$ and $\ell\left(I_{v}\right)=p_{2}$. In each iteration, clearly, at most one new interval length is added. Therefore, $I C(G) \leq I C(\mathcal{R}) \leq(m-1)+1=\lfloor(q+1) / 2\rfloor$.

Therefore, if a graph $G$ has $q$ maximal cliques, then $I C(G) \leq f(q)=\lfloor(q+1) / 2\rfloor \leq\lfloor(|V(G)|+1) / 2\rfloor$.
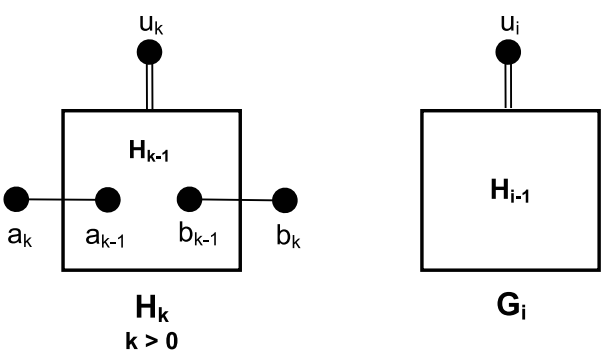

$\mathbf{G}_{\mathbf{i}}$

\section{Related problems}

It is clearly possible to assume, without loss of generality, that an interval graph or an interval order has an interval model in which all interval extreme points are integer numbers. In [18] (cf. [31]), Greenough discusses the problem of computing the minimum "width" of such models, that is, the problem of determining the minimum positive integer $r$ for which there exists an interval model of an order using only integer extreme points from the interval $[0, r]$. Related to this problem, we considered the interval count problem assuming that all interval extreme points are distinct and integer. Particularly, our question was to determine how the interval count of a graph (order) is affected when the interval models are assumed to have distinct integer extreme points. We showed in [9] that the interval count of a graph (order) is invariant under such an assumption. Motivated by both, this result and Greenough's discussion, we suggest the problem of determining the minimum positive integer $r$ for which there exists an interval model of an order realizing its interval count using only distinct integer extreme points from $[0, r]$.

There are also results regarding the interval lengths which are not directly concerned about minimizing their number. In [15], it is considered the problem of deciding whether there exists an interval model of a given interval graph such that each interval length is between $p$ and $q$ for a given pair of real numbers $1 \leq p \leq q$. Fishburn described a characterization by forbidden induced subgraphs of the graphs for which such a question has an affirmative answer. He proved that such a list is finite if and only if $p / q$ is a rational number. In this case, the characterization leads to an exponential-time $n^{O(p q)}$ recognition algorithm, where $n$ is the number of vertices of the graph. In [20], the author investigated this question restricted to the case in which all interval extreme points of the model are integer numbers and provided a polynomial-time $O\left(\min \left\{n^{3}, n^{2,5} \log (n(p+q))\right\}\right)$ recognition algorithm.

In [27], a more general question is studied. Let $G$ be an interval graph, and $\mathcal{C}$ be a set of constraints on the distances between interval extreme points of interval models of $G$. Each constraint is of the form $x-y \leq C_{x y}$, where $x$ and $y$ are interval extreme points, and $C_{x y}$ is a real number. 
The inequalities $\ell\left(I_{x}\right)-r\left(I_{y}\right) \leq 4$ and $r\left(I_{z}\right)-r\left(I_{w}\right) \leq-\sqrt{2}$ are examples of such constraints for vertices $x, y, z, w$. The problem consists in deciding whether there exists an interval model of $G$ satisfying all constraints in $\mathcal{C}$. A second problem comes from the previous one by requiring that each constraint involves only interval extreme points of the same vertex. Without loss of generality, in this case it is assumed that all constraints are in the form either $\ell\left(I_{v}\right)-r\left(I_{v}\right) \leq-L_{v}$ or $r\left(I_{v}\right)-\ell\left(I_{v}\right) \leq U_{v}$, where $v \in V(G)$, and $L_{v}, U_{v}$ are nonnegative real numbers. Equivalently, such constraints are written as $L_{v} \leq r\left(I_{v}\right)-\ell\left(I_{v}\right) \leq U_{v}$. Finally, a third problem is considered, originated from the previous one when $L_{v}=U_{v}$ for each constraint, i.e., each constraint is written in the form $r\left(I_{v}\right)-\ell\left(I_{v}\right)=L_{v}$, where $v \in V(G)$, and $L_{v}$ is a nonnegative real number. Pe'er and Shamir showed that this latter problem is NP-complete, and therefore so are the other two. On the other hand, restricted to the case of graphs that admit models having a unique ordering of its maximal cliques (up to reversion), they provided a polynomialtime algorithm to the first problem and, consequently, to the other two. They showed that for these graphs, the first problem can be reduced to that of deciding whether a system of inequalities is feasible, which can be decided in time $O\left(\min \left\{n^{3}, n^{2,5} \log (n C)\right\}\right)$, where $C$ is a number produced by the reduction.

\section{Concluding remarks}

The interval count is a basic problem regarding the wellknown class of interval graphs. Recognizing whether a graph is interval count one is a well-solved problem, for which there are several efficient algorithms. However, the problem of recognizing interval count $k, k \geq 2$, is widely open. When the problem is restricted to particular classes of graphs or orders, efficient algorithms for interval count $k$ are known. This is the case of extended-bull-free, generalizedthreshold, and almost- $K_{1,3}$-free graphs.

Intuitive conjectures have been proven not to hold. As examples, Graham's conjecture (the removal of a single vertex can decrease the interval count by at most one unit) was proven to hold in general only for graphs having interval count at most two. Similarly, the counterexamples for Trotter's conjecture (regarding the admissible lengths of the greatest size in a two-size representation) show that there exist unexpected "holes" in the set of admissible lengths.

Despite its fundamental importance for interval graphs, there are relatively few results on the interval count problem. We consider that this problem deserves more attention due to both its importance and the lack of improvement on the subject for more than twenty years.

Acknowledgements M.R. Cerioli and J.L. Szwarcfiter are partially supported by CAPES, CNPq, and FAPERJ.

\section{References}

1. Allen JF (1990) Maintaining knowledge about temporal intervals. Morgan Kaufmann, San Mateo

2. Allen JF, Kautz HA, Pelavin RN, Tenenberg JD (1991) Reasoning about plans. Morgan Kaufmann, San Mateo

3. Benzer S (1959) On the topology of the genetic fine structure. Proc Natl Acad Sci USA 45(11):1607-1620

4. Bogart KP, West DB (1999) A short proof that "proper = unit". Discrete Math 201(1-3):21-23

5. Bondy JA, Murty USR (2008) Graph theory. Springer, Berlin

6. Brandstädt A, Le VB, Szymczak T, Siegemund F Information system on graph class inclusions. http://wwwteo.informatik. uni-rostock.de/isgci/

7. Carrano AV (1988) Establishing the order to human chromosomespecific DNA fragments. In: Woodhead A, Barnhart B (eds) Biotechnology and the human genome. Plenum Press, New York, pp 37-50

8. Cerioli MR, Szwarcfiter JL (2006) Characterizing intersection graphs of substars of a star. Ars Comb 79:21-31

9. Cerioli MR, Oliveira FS, Szwarcfiter JL (2011) On counting interval lengths of interval graphs. Discrete Appl Math 159(7):532543

10. Coombs CH, Smith JEK (1973) On the detection of structures in attitudes and developmental processes. Psychol Rev 80:337-351

11. Corneil DG (2004) A simple 3-sweep LBFS algorithm for the recognition of unit interval graphs. Discrete Appl Math 138(3):371-379

12. Corneil DG, Kim H, Natarajan S, Olariu S, Sprague AP (1995) Simple linear time recognition of unit interval graphs. Inf Process Lett 55(2):99-104

13. de Figueiredo CMH, Meidanis J, de Mello CP (1995) A lineartime algorithm for proper interval graph recognition. Inf Process Lett 56(3):179-184

14. Deng X, Hell P, Huang J (1996) Linear-time representation algorithms for proper circular-arc graphs and proper interval graphs. SIAM J Comput 25(2):390-403

15. Fishburn PC (1985) Interval orders and interval graphs. Wiley, New York

16. Gardi F (2007) The Roberts characterization of proper and unit interval graphs. Discrete Math 307(22):2906-2908

17. Golumbic MC (2004) Algorithmic graph theory and perfect graphs, 2nd edn. Amsterdam, Elsevier

18. Greenough T (1974) The representation and enumeration of interval orders. PhD thesis, Darthmouth College

19. Hell P, Huang J (2005) Certifying lexbfs recognition algorithms for proper interval graphs and proper interval bigraphs. SIAM J Discrete Math 18(3):554-570

20. Isaak G (1993) Discrete interval graphs with bounded representation. Discrete Appl Math 33:157-183

21. Karp RM (1993) Mapping the genome: some combinatorial problems arising in molecular biology. In: STOC '93. ACM, New York, pp 278-285

22. Kendall DG (1969) Incidence matrices, interval graphs, and seriation in archaeology. Pac J Math 28:565-570

23. Leibowitz R (1978) Interval counts and threshold graphs. PhD thesis, Rutgers University

24. Leibowitz R, Assmann SF, Peck GW (1982) The interval count of a graph. SIAM J Algebr Discrete Methods 3(4):485-494

25. Nökel K (1991) Temporally distributed symptoms in technical diagnosis. Springer, Berlin

26. Papadimitriou CH, Yannakakis M (1979) Scheduling intervalordered tasks. SIAM J Comput 8(3):405-409

27. Pe'er I, Shamir R (1997) Realizing interval graphs with size and distance constraints. SIAM J Discrete Math 10(4):662-687 
28. Roberts FS (1969) Indifference graphs. In: Harary F (ed) Proof techniques in graph theory. Academic Press, San Diego, pp 139146

29. Roberts FS (1976) Discrete mathematical models with applications to social, biological, and environmental problems. Prentice Hall, New York

30. Skrien D (1984) Chronological orderings of interval graphs. Discrete Appl Math 8:69-83
31. Trotter WT (1988) Interval graphs, interval orders, and their generalizations. In: Applications of discrete mathematics. SIAM, Philadelphia, pp 45-58

32. Trotter WT (1992) Combinatorics and partially ordered sets. The Johns Hopkins University Press, Baltimore

33. Ward SA, Halstead RH (1990) Computation structures. MIT Press \& McGraw-Hill, Cambridge 